\title{
Efectos de la estimulación eléctrica babenular en la modulación de respuestas emocionales en ratas Wistar
}

\author{
María Laura Herrera ${ }^{1 *}$, Natalia Guisselle Rubio ${ }^{1}$, Juan Pablo Quintanilla ${ }^{1}$, Víctor Manuel Huerta ${ }^{1}$, Alejandro Osorio-Forero ${ }^{1}$, \\ Melissa Andre Cárdenas Molano ${ }^{1,2}$, Karen Corredor Páez ${ }^{1,3}, \mathrm{M}^{1}$. Valderrama ${ }^{1}$, Fernando Cárdenas ${ }^{1}$ \\ ${ }^{1}$ Universidad de los Andes, Bogotá, Colombia; ${ }^{2}$ Universidad Nacional de Colombia, Bogotá, Colombia; ${ }^{3}$ Centro de Investigaciones en \\ Biomodelos, CIBIOM, Bogotá, Colombia.
}

Recibido, agosto 17/2017

Concepto de evaluación, octubre $3 / 2017$

Aceptado, enero 26/2018
Referencia: Herrera, M. L., Rubio, N. G., Quintanilla, J. P., Huerta, V., Osorio-Forero, A., Cárdenas, M. A. N., Corredor, K., Valderrama, M. \& Cardenas, F. P. (2018). Efectos de la estimulación eléctrica habenular en la modulación de respuestas emocionales en ratas Wistar. Acta colombiana de Psicología, 21(2), 212-223. doi: http://www.dx.doi.org/10.14718/ACP.2018.21.2.10

Resumen

A pesar del amplio uso de la estimulación cerebral profunda para controlar patologías neurológicas y neuropsiquiátricas, su mecanismo de acción aún no es claramente conocido, y existen pocos estudios sistemáticos que relacionen la variación de parámetros de estimulación eléctrica (frecuencia, intensidad, duración del pulso) y la ejecución comportamental. La habénula es una estructura reguladora de respuestas emocionales diana en tratamientos para dolor crónico y depresión, pero la relación entre su estimulación crónica y el desempeño animal en pruebas conductuales no se ha establecido con claridad. Con el objetivo de evaluar el efecto emocional de la estimulación habenular crónica, en este estudio se utilizaron ratas Wistar que recibieron estimulación habenular a intensidad baja $(10-80 \mu \mathrm{A})$ o alta $(120-260 \mu \mathrm{A})$ y frecuencia baja $(80-150 \mathrm{~Hz})$ o alta $(240-$ $380 \mathrm{~Hz}$ ): BIBF-AIBF-BIAF-AIAF, durante 15 minutos a lo largo de tres días consecutivos. Al cuarto día, se hizo la evaluación en un laberinto elevado en cruz y en campo abierto. Los resultados indican un efecto de tipo ansiolítico en el tratamiento BIAF, en comparación con BIBF y AIBF (aumento del número de entradas, porcentaje de tiempo en brazos abiertos y de la distancia recorrida en ellos), efecto que no se explica por cambios en la locomotricidad (distancia recorrida en los brazos cerrados y la exploración en el campo abierto). Se concluye que el parámetro frecuencia posee mayor impacto sobre el efecto comportamental que la intensidad -lo que puede explicar algunos hallazgos paradójicos previos-, que los parámetros utilizados no poseen efecto ansiogénico, y que los efectos potencialmente ansiogénicos de la estimulación a baja frecuencia y el papel de los sistemas dopaminérgicos y serotoninérgicos encontrados deben ser estudiados en futuras investigaciones. Palabras clave: comportamiento emocional, estimulación eléctrica cerebral profunda, habénula, ratas.

\section{Effects of electrical stimulation of the habenula on the modulation of emotional responses in Wistar rats}

\begin{abstract}
Deep brain stimulation is a widely-used approach to the treatment of neurologic and neuropsychiatric diseases. However, its mechanisms remain unclear. There are few systematic studies relating variations on electrical stimulation parameters (frequency, intensity, pulse duration) and behavioral outcome. The habenula relates to emotional behavior and is a main target for chronic pain and depression stimulation treatment. The relation between habenular electrical stimulation and performance in behavioral tests has not been clearly defined. In order to assess the emotional effects of chronic habenular electrical stimulation, Wistar male rats were unilaterally implanted with electrodes aimed to the lateral habenula and assigned to low $(10-80 \mu \mathrm{A})$ or high $(120-260$ $\mu \mathrm{A})$ intensity and low $(80-150 \mathrm{~Hz})$ or high $(240-380 \mathrm{~Hz})$ frequency conditions: BIBF-AIBF-BIAF-AIAF. They received electrical stimulation 15 minutes/day for three consecutive days and on the fourth day were tested in the elevated plus maze and the open field. The results of these study show that BIAF stimulation has a possible anxiolytic-like effect when compared to BIBF and AIBF (increase in the percentage of open-arms time, entries into the open-arms and total-distance-run in the open-arms). This is not due to any changes in locomotion (total-distance-run and open field exploration). It is concluded that frequency is more important than intensity for behavioral modification. This could explain some previous inconsistent results. The data also suggest that these parameters of stimulation have no anxiogenic effects. The role for dopaminergic and serotonergic systems must be subsequently evaluated as well as potential anxiogenic-like effects of low frequency stimulation.
\end{abstract}

Key words: deep brain stimulation, emotional behavior, habenula, rats.

\footnotetext{
* Departamento de Ciencias Biológicas, +57(1) 3394999, lucarden@uniandes.edu.co
} 


\title{
Efeitos da estimulação elétrica habenular na modulaçáo de respostas emocionais em ratos Wistar
}

\begin{abstract}
Resumo
Apesar do amplo uso da estimulação cerebral profunda para controlar patologias neurológicas e neuropsiquiátricas, seu mecanismo de ação ainda não é claramente conhecido e existem poucos estudos sistemáticos que relacionem a variação de parâmetros de estimulação elétrica (frequência, intensidade, duração do pulso) e a execução comportamental. A habênula é uma estrutura reguladora de respostas emocionais específicas em tratamentos para dor crônica e depressão, mas a relação entre sua estimulação crônica e o desempenho animal em testes comportamentais não foi claramente estabelecida. Com o objetivo de avaliar o efeito emocional da estimulação habenular crônica, neste estudo foram utilizados ratos Wistar que receberam estimulação habenular de intensidade baixa $(10-80 \mu \mathrm{A})$ ou alta $(120-260 \mu \mathrm{A})$ e frequência baixa $(80-150 \mathrm{~Hz})$ ou alta $(240-380$ Hz): BIBF-AIBF-BIAF-AIAF, durante 15 minutos ao longo de três dias consecutivos. No quarto dia, foi feita a avaliação em um labirinto em cruz elevado e em campo aberto. Os resultados indicam um efeito de tipo ansiolítico no tratamento BIAF, em comparação com BIBF e AIBF (aumento do número de entradas, porcentagem de tempo em braços abertos e da distância percorrida neles), efeito que não se explica por mudanças na locomotividade (distância percorrida nos braços fechados e a exploração no campo aberto). Conclui-se que o parâmetro "frequência" tem mais impacto sobre o efeito comportamental do que a "intensidade" - o que pode explicar algumas descobertas paradoxais prévias -, que os parâmetros utilizados não tenham efeito ansiogênico, e que os efeitos potencialmente ansiogênicos da estimulação de baixa frequência e o papel dos sistemas dopaminérgicos e serotoninérgicos encontrados devem ser estudados em pesquisas futuras.

Palavras-chave: comportamento emocional, estimulação elétrica cerebral profunda, habênula, ratos.
\end{abstract}

\section{INTRODUCCIÓN}

La estimulación cerebral profunda (deep brain stimulation) es una de las técnicas de mayor desarrollo y potencial en los últimos años para el tratamiento futuro de diversas condiciones clínicas neurológicas y neuropsiquiátricas, incluyendo la enfermedad de Parkinson (Arocho-Quinones, Hammer, Bock \& Pahapill, 2017; Birchall et al., 2017; Borgonovo et al., 2017; Fukaya et al., 2017), las distonías y diskinesias (Almeida et al., 2017; Cif \& Coubes, 2017; Kim, Chang, Jung \& Chang, 2015; Lumsden, Kaminska, Ashkan, Selway \& Lin, 2017; Ostrem et al., 2017; Toda, Saiki, Nishida \& Iwasaki, 2016), la epilepsia (Cukiert \& Lehtimaki, 2017; Dalkilic, 2017; Klinger \& Mittal, 2016; Krishna, Sammartino, King, So \& Wennberg, 2016), el trastorno obsesivo compulsivo (Choudhury, Davidson, Viswanathan \& Strutt, 2017; Coenen et al., 2016; Dell'Osso, Cremaschi, Oldani \& Carlo, 2017; Mulders et al., 2016; Sturm et al., 2003), la ansiedad (Bewernick et al., 2010; Castelli et al., 2006; Chang et al., 2012; Clark et al., 2009), la agresividad (Faria, 2013; Golden et al., 2016; Harat, Rudas, Zielinski, Birska \& Sokal, 2015; Howland, 2013), el dolor crónico (Boadas-Vaello, Homs, Reina, Carrera \& Verdu, 2017; Li et al., 2017; Plotkin, 1982; Ray \& Burton, 1980), la obesidad (Dupre, Tomycz, Oh \& Whiting, 2015), la depresión (Accolla et al., 2016; Bergfeld et al., 2016; Bewernick et al., 2017; Birchall et al., 2017; Sourani, Eitan, Gordon \& Goelman, 2012) y la esquizofrenia (Agarwal,
Sarris, Herschman, Agarwal \& Mammis, 2016; Bakay, 2009; Nicolaidis, 2017; Salgado-Lopez et al., 2016), entre otras.

Los orígenes de la estimulación cerebral se remontan posiblemente al año 46 de nuestra era, cuando Scribonius Largus propuso colocar peces eléctricos (Torpedo nobiliana) en la cabeza de pacientes con migraña y epilepsia (Schwalb \& Hamani, 2008). Pero fue a finales del siglo XIX cuando Gustav Fritsch y Eduard Hitzig dieron inicio a la era de la estimulación eléctrica cerebral, al provocar contracciones musculares en perros anestesiados como consecuencia de la estimulación eléctrica de la corteza cerebral. Igualmente conocidos son los trabajos desarrollados a mediados del siglo XX por Wilder Penfield en humanos, los cuales llevaron al desarrollo de los mapas de representación motriz y sensorial en el córtex cerebral y que, sin lugar a dudas, constituyeron la piedra inicial para la actual estimulación cerebral profunda (Murrow, 2014).

En la actualidad, el primer reporte de uso de estimulación eléctrica cerebral en la edad moderna con fines terapéuticos proviene del trabajo de Lawrence Pool en 1948, quien trató exitosamente síntomas de depresión y anorexia en una paciente con enfermedad de Parkinson mediante el implante de un electrodo en el núcleo caudado (Rosenow, Mogilnert, Ahmed \& Rezai, 2004). Pero, posteriormente, a partir de mediados del siglo XX, la concientización sobre los graves efectos secundarios asociados a los tratamientos farmacológicos, tales como el L-Dopa, el avance tecnológico y el conocimiento en la fisiología de los ganglios basales (impulsado en gran medida por el estudio de modelos 
animales), fomentaron el uso extendido de la estimulación cerebral profunda para el tratamiento del Parkinson y de otros problemas motores asociados (Roth, Flashman, Saykin \& Roberts, 2001; Schwalb \& Hamani, 2008).

Otra de las áreas terapéuticas que se ha beneficiado por el uso de la estimulación eléctrica profunda, distinta al tratamiento de la sintomatología motriz, es el control del dolor crónico (Boccard, Pereira \& Aziz, 2015; Cruccu et al., 2016; Schwalb \& Hamani, 2008). Sin embargo, pese a la amplia difusión de la técnica, aún no son claros los mecanismos por los cuales funciona; y a pesar de que se ha encontrado que la estimulación de alta frecuencia puede tener un efecto superior a la de baja frecuencia, no es claro si el efecto se da por la depleción del neurotransmisor -asociada a la inducción de una alta tasa de disparo-o por el solo aumento de la liberación tónica de neurotransmisor. De hecho, la neurocirugía para el implante de electrodos de estimulación cerebral profunda se realiza con el paciente despierto, de forma tal que la efectividad de la estimulación se evalúa ajustando para cada caso los mejores parámetros de frecuencia, intensidad e intervalo inter-impulso. Y hasta donde se conoce, tampoco se han investigado sistemáticamente los efectos neurobiológicos a largo plazo sobre procesos como la up-regulation o la down-regulation de receptores, ni los cambios plásticos asociados al aumento (o disminución) de la actividad neuronal o posibles lesiones asociadas a los cambios de la dinámica sináptica en diferentes regiones del cerebro (Udupa \& Chen, 2015). Teniendo esto en cuenta, los estudios que permitan determinar los mecanismos por los cuales la estimulación cerebral profunda ejerce sus efectos se hacen completamente necesarios.

Por otra parte, utilizando la técnica de lesión electrolítica como herramienta para determinar el papel de diferentes estructuras en determinados comportamientos se ha demostrado que las lesiones de la habénula alteran diversos procesos, principalmente emocionales y comportamentales, como el procesamiento emocional del dolor (Li et al., 2017; Margolis \& Fields, 2016; Shelton, Becerra \& Borsook, 2012), las situaciones de refuerzo (Baker, Raynor, Francis \& Mizumori, 2017; Borsook et al., 2016; Jean-Richard Dit \& McNally, 2014), el proceso de plasticidad sináptica -en un protocolo de depresión a largo plazo- (Lecourtier et al., 2006) e, incluso, la emergencia de comportamientos relacionados con la adicción (Baldwin, Alanis \& Salas, 2011; Lecca, Meye \& Mameli, 2014; Velasquez, Molfese \& Salas, 2014; Yadid, Gispan \& Lax, 2013).

Uno de los primeros hallazgos en el estudio de lesiones habenulares indicó que la lesión de algunas vías eferentes de la habénula (principalmente, el fascículo retroflexo) ocasiona un aumento de ansiedad y locomotricidad en ratas, así como un incremento de los niveles plasmáticos de corticosterona y los comportamientos de acicalamiento (Murphy, DiCamillo, Haun \& Murray, 1996). Igualmente, resulta interesante que tras la lesión habenular se pierde la capacidad de reaccionar adecuadamente al estrés -aunque algunos autores han encontrado resultados contradictorios(Amat et al., 2001; Hennigan, D'Ardenne \& McClure, 2015; Thornton \& Bradbury, 1989). Sin embargo, los resultados aparentemente contradictorios podrían explicarse si se toma en consideración que la habénula posee un rol importante en la evaluación de las posibilidades de refuerzo de diversos estímulos (Bromberg-Martin \& Hikosaka, 2011), y porque, de hecho, la activación de la habénula conduce a cambios en la actividad dopaminérgica y serotoninérgica (Yang, Hu, Xia, Zhang \& Zhao, 2008), por lo que la activación diferencial de cada uno de esos sistemas podría darse en función de su patrón de actividad.

Con respecto a la ansiedad, se ha demostrado que la inactivación de la habénula ocasiona una reducción de los niveles de ansiedad en el laberinto en cruz elevado (Gill, Ghee, Harper \& See, 2013), pero hasta la fecha no se tienen reportes del efecto del incremento de la actividad habenular sobre este tipo de comportamientos. Así, teniendo lo anterior en cuenta, el objetivo de este trabajo fue determinar si diferentes patrones de estimulación de la habénula lateral conducen a alteraciones en la expresión de comportamientos asociados a la ansiedad y la locomotricidad.

Para tal fin, y buscando la realización de un estudio sistemático, se seleccionaron dos parámetros de análisis: la frecuencia y la intensidad de la estimulación. Y cada uno de estos, teniendo en cuenta reportes previos sobre estimulación cerebral profunda (Arocho-Quinones et al., 2017; Kim et al., 2016; Li et al., 2016), fue dividido en dos niveles: (a) frecuencia: alto y bajo, con un punto de corte de $150 \mathrm{~Hz}$; y (b) intensidad: alto y bajo, con un punto de corte de 100 uA (Yeomans, 1990).

\section{MÉTODO}

\section{Diseño}

Se utilizó un diseño experimental con cuatro grupos experimentales y un grupo control, sin medidas repetidas. Los grupos experimentales recibieron estimulación eléctrica cerebral profunda unilateral en la habénula, con variación en la intensidad -baja $(10-80 \mu \mathrm{A})$ o alta $(120-260 \mu \mathrm{A})-\mathrm{y}$ en la frecuencia -baja $(80-150 \mathrm{~Hz})$ o alta $(240-380 \mathrm{~Hz})-$.

\section{Sujetos}

Se utilizaron 26 ratas Wistar machos, con pesos entre 250 y 350 g, provenientes de una colonia exocriada del 
Charles River Institute, mantenidas en el Laboratorio de Neurociencia y Comportamiento de la Universidad de los Andes. Los animales se alojaron en cajas hogar en condiciones controladas de iluminación (ciclo luz-oscuridad de 12:12 h; luz iniciada a las 05:00 p. m.), temperatura (23 ${ }^{\circ} \mathrm{C} \pm 2$ ), humedad relativa mantenida al $57 \%$, con libre acceso a agua y comida durante todo el experimento y con atenuación de sonidos externos. Todos los sujetos experimentales fueron manipulados durante diez minutos al día desde tres días antes del inicio de los experimentos con el fin de familiarizarlos con el experimentador, los procesos y las salas experimentales.

\section{Aparatos}

Estimulación cerebral profunda. Para la estimulación cerebral se utilizó un estimulador de pulsos S88X (GRASS) con cuatro parámetros de control de pulso (frecuencia, duración, repetitividad en trenes e intervalo inter-impulso) para dos salidas independientes de estimulación por corriente.

Laberinto en cruz elevado. Para evaluar los niveles de ansiedad de los animales se utilizó el laberinto en cruz elevado, un artefacto de madera con dos brazos de $1 \mathrm{~m}$ cruzados perpendicularmente entre sí, dos de ellos encerrados por paredes de $50 \mathrm{~cm}$ de altura (brazos cerrados) y dos descubiertos (brazos abiertos), con un borde de $1 \mathrm{~cm}$ de altura para evitar la caída de los animales. El aparato completo se encuentra elevado a una altura de $50 \mathrm{~cm}$ del suelo. Los experimentos fueron grabados y digitalizados para su posterior análisis. Para el registro comportamental, cada brazo fue dividido virtualmente en cuadrados de $10 \mathrm{~cm}$.

Campo abierto. El campo abierto consiste en una caja cúbica de acrílico obscuro de $60 \mathrm{~cm}$ de lado, sin la tapa superior. Los experimentos fueron grabados y digitalizados para su posterior registro. Para el registro comportamental, el piso del campo abierto fue virtualmente dividido en cuadrados de $10 \mathrm{~cm}$ de lado.

\section{Procedimiento}

Los sujetos fueron asignados aleatoriamente a uno de cinco grupos para recibir estimulación cerebral profunda en la habénula con diferentes parámetros de frecuencia y de intensidad. Las características de estimulación de los grupos comprendían intensidades bajas $(10-80 \mu \mathrm{A})$ y altas $(120-260$ $\mu \mathrm{A})$, y frecuencias bajas $(80-150 \mathrm{~Hz})$ y altas $(240-380 \mathrm{~Hz})$. De esa forma, se conformaron grupos de baja intensidad y baja frecuencia (BIBF, $n=5$ ), alta intensidad y baja frecuencia (AIBF, $n=5$ ), baja intensidad y alta frecuencia
(BIAF, $\mathrm{n}=5$ ), alta intensidad y alta frecuencia (AIAF, $\mathrm{n}=5$ ), $\mathrm{y}$ un grupo control $(\mathrm{n}=6)$ sometido a los mismos procedimientos, pero sin recibir ningún tipo de estimulación. En la Tabla 1 se presenta la asignación de sujetos.

Tabla 1

Asignación de sujetos a grupos experimentales

\begin{tabular}{lll}
\hline \multicolumn{1}{c}{ Grupo } & \multicolumn{1}{c}{ Intensidad } & \multicolumn{1}{c}{ Frecuencia } \\
\hline BIBF, $\mathrm{n}=5$ & Baja $(10-80 \mu \mathrm{A})$ & Baja $(80-150 \mathrm{~Hz})$ \\
AIBF, $\mathrm{n}=5$ & Alta $(120-260 \mu \mathrm{A})$ & Baja $(80-150 \mathrm{~Hz})$ \\
BIAF, $\mathrm{n}=5$ & Baja $(10-80 \mu \mathrm{A})$ & Alta $(240-380 \mathrm{~Hz})$ \\
AIAF, $\mathrm{n}=5$ & Alta $(120-260 \mu \mathrm{A})$ & Alta $(240-380 \mathrm{~Hz})$ \\
Grupo control, $\mathrm{n}=6$ & - & - \\
\hline
\end{tabular}

Para la estimulación eléctrica crónica de la habénula se colocaron electrodos intracerebrales mediante cirugía estereotáxica tradicional. En detalle, los animales fueron anestesiados con una inyección intraperitoneal de pentobarbital (Penthal, $64.8 \mathrm{mg} / \mathrm{kg}$ ) y puestos en un aparato estereotáxico, como anestésico local se aplicó lidocaína (2\%). Durante toda la cirugía se monitorearon los signos vitales y se mantuvo la temperatura con una manta térmica. Se retiró el cuero cabelludo y con la ayuda de un motortool se abrió un agujero de $0.5 \mathrm{~mm}$ en el cráneo expuesto, a través del cual se insertó unilateralmente el electrodo, con una inclinación de $20^{\circ}$. La ubicación final del electrodo fue: AP: $-3.6 \mathrm{~mm}$, ML: $0.7 \mathrm{~mm}$ y DV: $5.0 \mathrm{~mm}$, de acuerdo con el atlas de Paxinos y Watson (2006), tomando el punto bregma como referencia. El electrodo fue fijado al cráneo por medio de dos tornillos de anclaje y acrílico dental. Terminada la cirugía, los animales recibieron tratamiento con antiinflamatorio/analgésico (Meloxicám, $1 \mathrm{mg} / \mathrm{kg}$ ) y antibiótico (Enrofloxacina, $10 \mathrm{mg} / \mathrm{kg}$ ). Todos los animales tuvieron un período de recuperación de seis días después de la cirugía.

Para la realización de la estimulación, cada animal era retirado de su caja hábitat y colocado en la caja de estimulación. Luego de un periodo de familiarización de un minuto, se iniciaba la estimulación a la combinación de frecuencia e intensidad previamente asignada al sujeto, dependiendo del grupo al cual correspondía. La estimulación tenía una duración de 15 minutos continuos por día y fue realizada durante tres días consecutivos. Veinticuatro horas después de la última estimulación, los animales fueron transportados a las salas experimentales para su valoración comportamental. Debido a la gran sensibilidad del laberinto en cruz elevado para cambios del estado de ansiedad de los animales, esta prueba fue realizada siempre antes del campo abierto. En la Figura 1 se presenta un esquema del procedimiento realizado.

En la fase de evaluación comportamental, cada animal fue colocado en el cuadrado central del laberinto, con el hocico dirigido hacia uno de los brazos cerrados, y se le permitió 


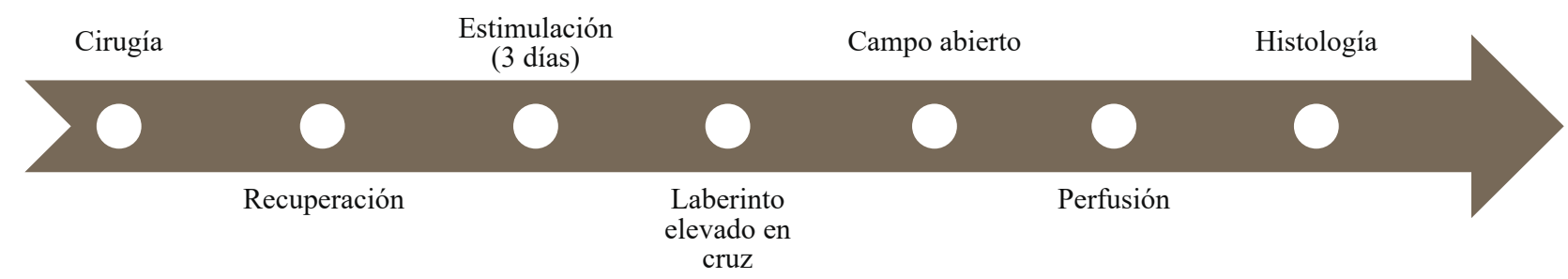

Figura 1. Procedimiento experimental.

explorar libremente durante cinco minutos. Durante este periodo, se cuantificó el número de entradas y tiempo de permanencia en cada tipo de brazo y en el área central. Se registraron, además, las frecuencias y tiempos de duración de comportamientos, como el levantarse, el estiramiento y el mirar hacia abajo -por el borde de los brazos abiertos-.

Finalizado el tiempo de exploración en el laberinto en cruz elevado, el animal fue llevado a otra sala experimental para su evaluación en el campo abierto. Luego, se ubicó a cada sujeto en el centro del campo de cara a una de las paredes del mismo y se permitió que explorara libremente durante cinco minutos. Se contabilizó el tiempo que permanecía en la periferia (definida como un corredor de 10 $\mathrm{cm}$ de ancho desde cualquiera de las paredes) y en el centro del campo. Siempre, después de finalizar la exploración de cada animal en cada test comportamental, el aparato fue limpiado con alcohol al $10 \%$ para evitar pistas olfativas.

Finalizada la evaluación comportamental, los animales fueron anestesiados con una mezcla de ketamina/xilacina $(90 \mathrm{mg} / \mathrm{kg}+10 \mathrm{mg} / \mathrm{kg}$ ) y perfundidos vía transcardiaca con $300 \mathrm{ml}$ de solución salina $(0.9 \%)$, seguida por 300 $\mathrm{ml}$ de paraformaldehido (4\%). Finalmente, los cerebros fueron extraídos, almacenados en paraformaldehido (4\%) durante cuatro días y procesados para su uso histológico con el fin de verificar la ubicación de los electrodos, para lo cual se realizaron cortes coronales de $30 \mu \mathrm{m}$ de grosor en un vibrátomo (VIBRATOME 1500). Estos cortes fueron posteriormente marcados con coloración de Nissl por violeta de cresil. Los datos correspondientes a los animales que mostraron ubicaciones del punto de estimulación diferentes a la deseada fueron retirados del estudio.

\section{Consideraciones éticas}

Todos los procedimientos realizados en este trabajo fueron aprobados por el Comité Institucional de Cuidado y Uso de Animales de Laboratorio (CICUAL) de la Universidad de los Andes y estuvieron en consonancia con las normas éticas y legales exigidas para la investigación con animales de laboratorio en Colombia (Ley 84 de 1989 y Resolución No. 8430 de 1993 del Ministerio de Salud). Adicionalmente, se respetaron los acuerdos de la declaración universal de los derechos de los animales, proclamada por la Liga Internacional de los Derechos del Animal, Ginebra, Suiza (1989), y los principios éticos de la experimentación animal enunciados por el International Council for Laboratory Animal Science (ICLAS).

\section{Análisis de datos}

Los tests de normalidad (Shapiro-Wilk) fueron aprobados para todas las mediciones, lo cual permitió la realización de un Análisis de Varianza de una vía. Cuando fue necesario, se realizó el análisis post hoc, utilizando la prueba de Tukey. En todos los casos, se estableció un alfa de .05.

\section{RESULTADOS}

A continuación, se presentan los resultados del estudio, iniciando con los hallazgos histológicos, y luego con los comportamentales tanto emocionales como de motricidad.

\section{Histología}

En la Figura 2 se muestra la ubicación de los electrodos. Los animales que tuvieron electrodos en lugares diferentes al deseado fueron retirados del experimento.

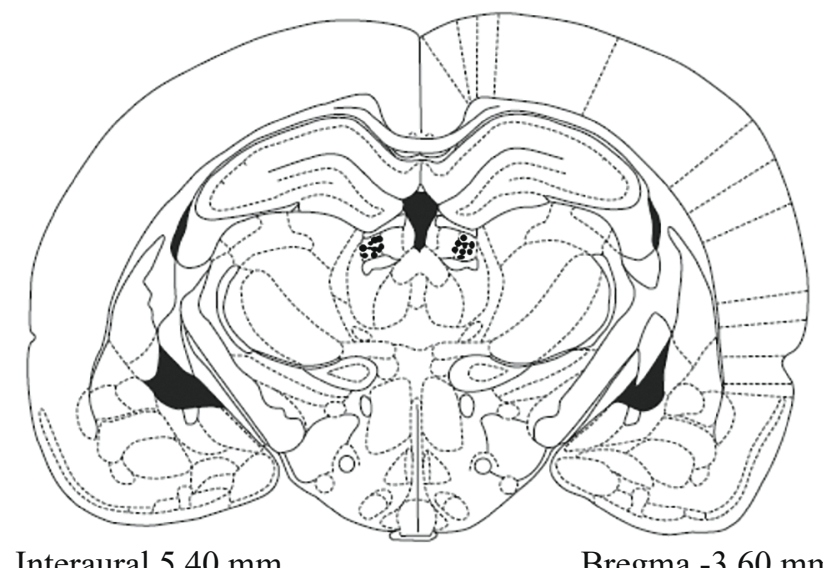

Interaural $5.40 \mathrm{~mm}$

Bregma $-3.60 \mathrm{~mm}$

Figura 2. Puntos de llegada de los electrodos. Adaptado de Paxinos y Watson (2006). 


\section{Laberinto en cruz elevado}

Con respecto al número de entradas a los brazos abiertos, elANOVA mostró diferencias significativas entre los efectos de los tipos de estimulación $\left(\mathrm{F}_{[4,21]}=6.71 ; \mathrm{p}<.001\right)$; y la comparación post hoc de las medias de los grupos (Tukey) evidenció que los animales del grupo BIAF entraron más a los brazos abiertos del laberinto que los animales de los grupos AIBF y BIBF $(\mathrm{p}<.05)$. No se encontró efecto sobre la cantidad de entradas a los brazos cerrados (Figura 3-B), $\left(\mathrm{F}_{[4,21]}=.91 ; \mathrm{p}=.475\right)$.

Con respecto al porcentaje de entradas a los brazos abiertos (Figura 3-A), el ANOVA mostró diferencias significativas entre los efectos de la estimulación $\left(\mathrm{F}_{[4,21]}=3.06\right.$; $\mathrm{p}=.039)$; y la comparación post hoc de las medias de los grupos (Tukey) evidenció que los animales del grupo BIAF entraron más a los brazos abiertos del laberinto que los animales de los grupos AIBF $(\mathrm{p}<.05)$.

Adicionalmente, el ANOVA mostró un efecto de la estimulación sobre el porcentaje de tiempo de permanencia en los brazos abiertos $\left(\mathrm{F}_{[4,21]}=4.43 ; \mathrm{p}=.01\right.$, véase Figura 3-C); y la comparación post hoc de las medias de los grupos (Tukey) mostró que los animales del grupo BIAF permanecieron más en los brazos abiertos del laberinto que los animales de los grupos AIBF y BIBF $(\mathrm{p}<.05)$. Con respecto a la distancia toral recorrida dentro del brazo abierto (Figura 3-D), el ANOVA evidenció un efecto significativo de la estimulación $\left(\mathrm{F}_{[4,21]}=4.88 ; \mathrm{p}=.01\right)$; y la comparación post hoc de las medias de los grupos mostró que los animales del grupo BIAF recorrieron mayor distancia que los animales de los grupos AIBF y BIBF $(\mathrm{p}<.05)$ (véase Tabla 2 para los resultados estadísticos de todas las medidas).

\section{Campo abierto}

La ejecución de los diferentes sujetos en el campo abierto mostró que no hubo diferencias significativas en la locomoción -medida en términos de la distancia total recorrida a partir del número total de cruzamientos realizados- $\left(\mathrm{F}_{[4,21]}=.57 ; \mathrm{p}=.642\right) ; \mathrm{y}$ tampoco se encontraron

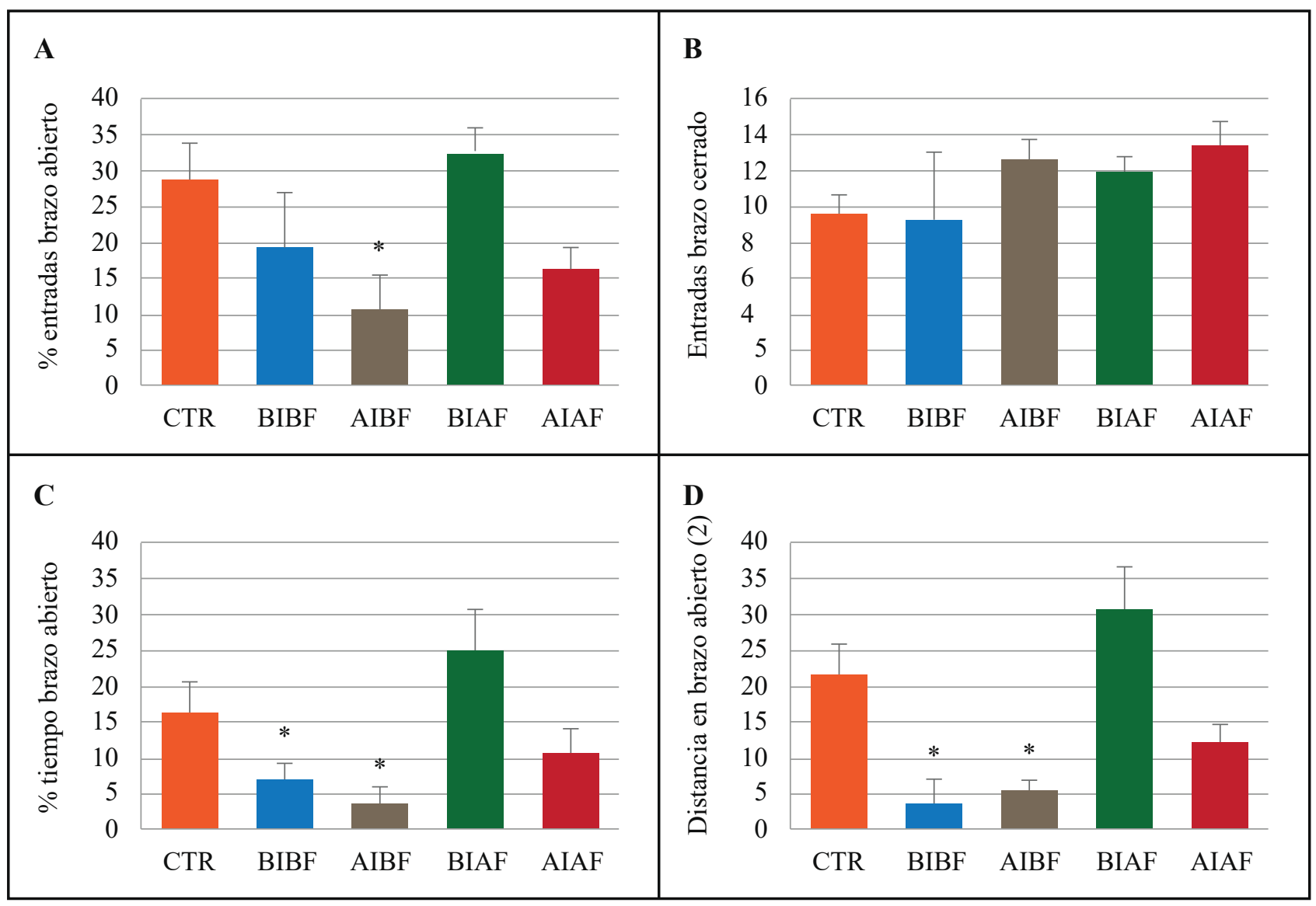

Figura 3. (A) Entradas a los brazos abiertos del laberinto en cruz elevado. (B) Entradas a los brazos cerrados. (C) Porcentaje de tiempo de permanencia en los brazos abiertos. (D) Distancia total recorrida dentro del brazo abierto. $\left.{ }^{*}\right)$ diferencia con respecto al grupo BIAF. $\mathrm{p}<.05$. 
Tabla 2

Resultados estadísticos para los comportamientos en el laberinto en cruz elevado

\begin{tabular}{lcc}
\hline \multicolumn{1}{c}{ Comportamiento } & $\mathrm{F}[4,21]$ & $\mathrm{p}$ \\
\hline Entradas brazo abierto & 6.709 & $<.001^{*}$ \\
Porcentaje de entradas al brazo abierto & 3.058 & $.039^{*}$ \\
Porcentaje de tiempo en brazo abierto & 4.430 & $.009^{*}$ \\
Distancia recorrida en brazo abierto & 4.880 & $.006^{*}$ \\
Entradas brazo cerrado & 0.912 & .475 \\
Porcentaje de tiempo en brazo cerrado & 4.430 & $.009^{*}$ \\
Distancia recorrida en brazo cerrado & 0.590 & .672 \\
Porcentaje de tiempo en el centro & 2.420 & .080 \\
Tiempo de acicalamiento & 1.191 & .343 \\
\hline
\end{tabular}

Tabla 3

Resultados estadísticos para los comportamientos en el campo abierto

\begin{tabular}{lcc}
\hline \multicolumn{1}{c}{ Comportamiento } & $\mathrm{F}[4,21]$ & $\mathrm{p}$ \\
\hline Entradas área central & 0.853 & .051 \\
Distancia total recorrida & 0.569 & .642 \\
Porcentaje de tiempo en área central & 0.867 & .501 \\
Cantidad de levantamientos & 1.528 & .232 \\
Tiempo de levantamiento & 1.517 & .235 \\
Frecuencia de acicalamiento & 0.590 & .674 \\
Tiempo de acicalamiento & 1.440 & .257 \\
Frecuencia de congelamiento & 0.570 & .688 \\
Tiempo de congelamiento & 0.365 & .831 \\
\hline
\end{tabular}

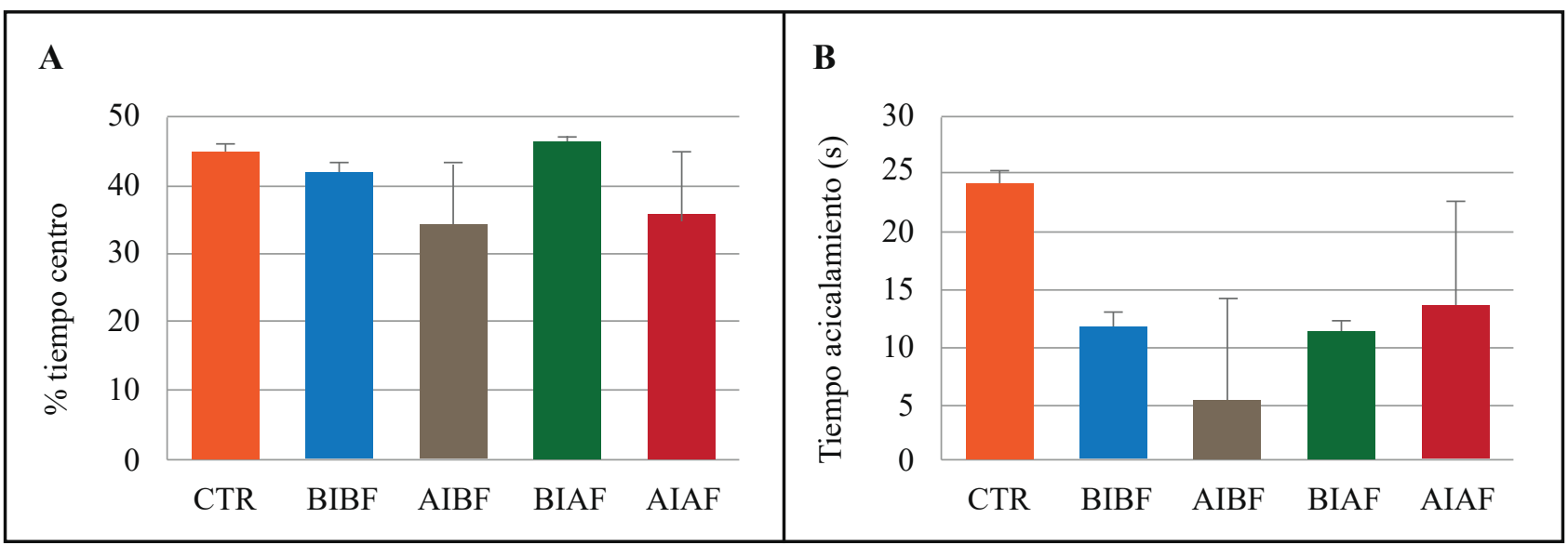

Figura 4. (A) Porcentaje de permanencia en el área central del campo abierto. (B) Tiempo de limpieza en el campo abierto.

diferencias significativas para el porcentaje de tiempo de permanencia en el centro ni para el tiempo empleado en acicalamiento $\left(\mathrm{F}_{[4,21]}=.87 ; \mathrm{p}=.501, \mathrm{~F}_{[4,21]}=1.44 ; \mathrm{p}=.26\right.$, respectivamente) (véase Figura 4 y Tabla 3 para los resultados estadísticos de todas las medidas).

\section{DISCUSIÓN}

En el presente estudio se evaluó el efecto de la estimulación eléctrica de la habénula lateral sub-crónica sobre los niveles de ansiedad y la respuesta locomotriz en ratas Wistar. Luego de la estimulación eléctrica de la habénula lateral por quince minutos durante tres días, se hizo una evaluación en el laberinto en cruz elevado y en un campo abierto.

La habénula lateral ha sido vinculada con numerosos procesos emocionales y cognitivos, pero a pesar de que en diversos estudios se ha utilizado la estimulación eléctrica de esta estructura como metodología, aún no hay consenso sobre el tipo de estimulación más efectiva (Baker et al., 2016; 
Baker, Oh, Kidder \& Mizumori, 2015; Chan et al., 2017; Hikosaka, Sesack, Lecourtier \& Shepard, 2008; Hong \& Hikosaka, 2008; Lecourtier, Neijt \& Kelly, 2004; Moreines, Owrutsky \& Grace, 2017; Ootsuka \& Mohammed, 2015; Zhao, Zhang, Yang \& Rusak, 2015). Por ejemplo, algunos autores, como Li et al. $(2016 ; 2017)$, reportan resultados positivos con estimulación eléctrica a una frecuencia de $130 \mathrm{~Hz}$ y $150 \mu$ A de intensidad (equivalente al grupo AIBF en este estudio), en tanto que, con frecuencias menores (como de $80 \mathrm{~Hz}$ ), no encuentran estos resultados a la misma intensidad (equivalente al mismo grupo en este trabajo).

En este sentido, los resultados expuestos indican que la estimulación eléctrica de la habénula a alta frecuencia (240-380 Hz) induce claros efectos comportamentales al disminuir las respuestas de ansiedad en el laberinto en cruz elevado, y que la estimulación de la misma estructura con bajas frecuencias $(80-150 \mathrm{~Hz})$ no induce cambios significativos. Estos datos contrastan con los reportes de Heldt y Ressler (2006) y de Gill et al. (2013), quienes describieron cambios emocionales asociados con lesiones de la habénula, en donde mencionan la reducción de las respuestas de ansiedad en ratas con inactivación de la habénula. Sus resultados, pese a utilizar otra metodología de estudio, permiten determinar que la habénula tiene un rol importante en la generación y expresión de reacciones emocionales. Tal vez la estimulación a la que fueron sometidos los animales del presente estudio ocasionó un efecto inhibitorio sobre las eferencias habenulares, ya sea por un fenómeno de depleción de neurotransmisores o por disminución en la expresión de receptores en las estructuras postsinápticas. Desafortunadamente, estos resultados no permiten determinar el mecanismo causante de dicho efecto.

Por otra parte, Song et al. (2017) reportaron recientemente que la lesión completa de la habénula lateral facilita el aprendizaje de la respuesta de evitación activa y la extinción del miedo, datos que parecerían ir en contra de los hallazgos de este estudio; sin embargo, podría proponerse que la estimulación realizada en la habénula posea efectos a largo plazo (quizá mediados por procesos de desensibilización de receptores en estructuras diana) o bien que la estimulación realizada genere un estado de "fatiga" neuronal (posiblemente por depleción de neurotransmisores) que permanece por algunos minutos después de finalizada la estimulación, y que simularía el efecto de la lesión realizada por Song et al. (2017). En cualquier caso, se requiere de la realización de nuevos experimentos para determinar el mecanismo en cuestión.

Los hallazgos reportados, inducidos por la estimulación de la habénula, parecen estar restringidos a la esfera emocional, pues no se encontraron cambios en la función motriz, evaluada tanto en el laberinto en cruz elevado mediante el análisis de la distancia total recorrida en el brazo cerrado, como en el campo abierto. En este sentido, los datos están en consonancia con los reportados por varios autores con respecto a aumentos de la función motriz asociados a lesiones de la habénula (Han, Jin, Song, Wang \& Zhao, 2014; Murphy et al., 1996; Wickens \& Thornton, 1996). Adicionalmente, no se encontraron alteraciones en el comportamiento de acicalamiento en el laberinto en cruz elevado ni en el campo abierto, a pesar de que, de acuerdo con los hallazgos de Murphy et al. (1996), se esperaría una disminución de los mismos.

Por otra parte, existen numerosos reportes que correlacionan cambios en los niveles cerebrales de serotonina (principalmente en la amígdala y en el córtex prefrontal) con un aumento de las respuestas de tipo ansioso en el laberinto en cruz elevado (Andersen \& Teicher, 1999; Dos, de Andrade \& Graeff, 2010; Faria et al., 2006; John \& Currie, 2012; Lin \& Parsons, 2002; Maisonnette, Morato \& Brandao, 1993; Moraes, Bertoglio \& Carobrez, 2008; Motta, Maisonnette, Morato, Castrechini \& Brandao, 1992; Setem, Pinheiro, Motta, Morato \& Cruz, 1999), pero, debido a que la activación de la habénula posee un efecto facilitador sobre la liberación de serotonina (Lim et al., 2015; Pobbe \& Zangrossi, 2010; Rolls, 2017; Vadovicova, 2014; Yang et al., 2008), sería factible suponer que el efecto de aumento del tiempo de permanencia en los brazos abiertos del laberinto en cruz elevado, así como el aumento de la distancia total recorrida dentro de ese mismo tipo de brazos, sea un efecto de adaptación mediado por serotonina.

Finalmente, pese a no haber encontrado diferencias significativas con relación al grupo control, es evidente que los grupos que recibieron estimulación de baja frecuencia mostraron una marcada disminución en la exploración del brazo abierto en términos del porcentaje de permanencia $(\bar{x}[\mathrm{CTR}]=16.7 ; \bar{x}[\mathrm{BIBF}]=6.2 ; \bar{x}[\mathrm{AIBF}]=3.6)$, número de entradas $(\bar{x}[\mathrm{CTR}]=4.0 ; \bar{x}[\mathrm{BIBF}]=1.2 ; \bar{x}[\mathrm{AIBF}]=1.6)$ $\mathrm{y}$ distancia total recorrida en ellos $(\bar{x}[\mathrm{CTR}]=21.7$; $\bar{x}[\mathrm{BIBF}]=3.6 ; \bar{x}[\mathrm{AIBF}]=5.0$ ). Para futuras investigaciones se recomienda la exploración de frecuencias menores con el fin de confirmar si podrían aumentar tales diferencias y constituir un posible efecto ansiogénico.

\section{REFERENCIAS}

Accolla, E. A., Aust, S., Merkl, A., Schneider, G. H., Kuhn, A. A., Draganski, B. (2016). Deep brain stimulation of the posterior gyrus rectus region for treatment resistant depression. Journal of Affective Disorders, 194, 33-37. Doi: https:// doi:10.1016/j.jad.2016.01.022 
Agarwal, P., Sarris, C. E., Herschman, Y., Agarwal, N., \& Mammis, A. (2016). Schizophrenia and neurosurgery: A dark past with hope of a brighter future. Journal of Clinical Neuroscience, 34, 53-58. Doi: https://doi:10.1016/j. jocn.2016.08.009

Almeida, L., Martinez-Ramirez, D., Ahmed, B., Deeb, W., Jesus, S., \& Okun, M. S. (2017). A pilot trial of square biphasic pulse deep brain stimulation for dystonia: The BIP dystonia study. Movement Disorders Journal, 32, 615-618. Doi: https://doi:10.1002/mds.26906

Amat, J., Sparks, P. D., Matus-Amat, P., Griggs, J., Watkins, L. R., \& Maier, S. F. (2001). The role of the habenular complex in the elevation of dorsal raphe nucleus serotonin and the changes in the behavioral responses produced by uncontrollable stress. Brain Research Bulletin, 917, 118-126. Doi: https://doi.org/10.1016/S0006-8993(01)02934-1

Andersen, S. L., \& Teicher, M. H. (1999). Serotonin laterality in amygdala predicts performance in the elevated plus maze in rats. Neuroreport Journal, 10, 3497-3500.

Arocho-Quinones, E. V., Hammer, M. J., Bock, J. M., \& Pahapill, P. A. (2017). Effects of deep brain stimulation on vocal fold immobility in Parkinson's disease. Surgical Neurology International, 8, 22. Doi: https://doi:10.4103/21527806.200580.e

Bakay, R. A. (2009). Deep brain stimulation for schizophrenia. Stereotactic and functional neurosurgery, 87, 266. Doi: https://doi.org/10.1159/000225980

Baker, P. M., Jhou, T., Li, B., Matsumoto, M., Mizumori, S. J., ... Vicentic, A. (2016). The Lateral Habenula Circuitry: Reward Processing and Cognitive Control. Journal of Neuroscience, 36, 11482-11488. Doi: https://doi.org/10.1523/ JNEUROSCI.2350-16.2016

Baker, P. M., Oh, S. E., Kidder, K. S., \& Mizumori, S. J. (2015). Ongoing behavioral state information signaled in the lateral habenula guides choice flexibility in freely moving rats. Frontiers in Behavioral Neuroscience, 9, 295. Doi: https:// doi:10.3389/fnbeh.2015.00295

Baker, P. M., Raynor, S. A., Francis, N. T., \& Mizumori, S. J. (2017). Lateral habenula integration of proactive and retroactive information mediates behavioral flexibility. Neuroscience, 345, 89-98. Doi: https://doi:10.1016/j.neuroscience.2016.02.010

Baldwin, P. R., Alanis, R., \& Salas, R. (2011). The Role of the Habenula in Nicotine Addiction. Journal of Addiction Research \& Therapy, SI. Doi:https://doi: 10.4172/2155-6105. S1-002

Bergfeld, I. O., Mantione, M., Hoogendoorn, M. L., Ruhe, H. G., Notten, P., ... Denys, D. (2016). Deep Brain Stimulation of the Ventral Anterior Limb of the Internal Capsule for Treatment-Resistant Depression: A Randomized Clinical Trial. JAMA Psychiatry, 73, 456-464. Doi: https:// doi: $10.1017 / \mathrm{S} 0033291717000113$

Bewernick, B. H., Hurlemann, R., Matusch, A., Kayser, S., Grubert, C., ... Schlaepfer, T (2010). Nucleus accumbens deep brain stimulation decreases ratings of depression and anxiety in treatment-resistant depression. Biological Psychiatry, 67, 110-116. Doi: https://doi:10.1016/j.biopsych.2009.09.013

Bewernick, B. H., Kayser, S., Gippert, S. M., Switala, C., Coenen, V. A., \& Schlaepfer, T. E. (2017). Deep brain stimulation to the medial forebrain bundle for depressionlong-term outcomes and a novel data analysis strategy. Brain Stimulation, 10, 664-671. Doi: https://doi:10.1016/j. brs.2017.01.581

Birchall, E. L., Walker, H. C., Cutter, G., Guthrie, S., Joop, A., ... Amara, A. W. (2017). The effect of unilateral subthalamic nucleus deep brain stimulation on depression in Parkinson's disease. Brain Stimulation, 10, 651-656. Doi: https:// doi:10.1016/j.brs.2016.12.014

Boadas-Vaello, P., Homs, J., Reina, F., Carrera, A., \& Verdu, E. (2017). Neuroplasticity of Supraspinal Structures Associated with Pathological Pain. Anatomical Record, (Hoboken). Doi: https://doi:10.1002/ar.23587

Boccard, S. G., Pereira, E. A., \& Aziz, T. Z. (2015). Deep brain stimulation for chronic pain. Journal of Clinical Neuroscience, 22, 1537-1543. Doi: https://doi:10.1016/j. jocn.2015.04.005

Borgonovo, J., Allende-Castro, C., Laliena, A., Guerrero, N., Silva, H., \& Concha, M. L. (2017). Changes in neural circuitry associated with depression at pre-clinical, premotor and early motor phases of Parkinson's disease. Parkinsonism and Related Disorders, 35, 17-24. Doi: https:// doi:10.1016/j.parkreldis.2016.11.009

Borsook, D., Linnman, C., Faria, V., Strassman, A. M., Becerra, L., \& Elman, I. (2016). Reward deficiency and anti-reward in pain chronification. Neuroscience \& Biobehavioral Reviews, 68, 282-297. Doi: https://doi:10.1016/j.neubiorev.2016.05.033

Bromberg-Martin, E. S., \& Hikosaka, O. (2011). Lateral habenula neurons signal errors in the prediction of reward information. Nature Neuroscience, 14, 1209-1216. Doi: https:// doi:10.1038/nn.2902

Castelli, L., Perozzo, P., Zibetti, M., Crivelli, B., Morabito, U., Lanotte, M., ... Lopiano, L. (2006). Chronic deep brain stimulation of the subthalamic nucleus for Parkinson's disease: effects on cognition, mood, anxiety and personality traits. European Neurology, 55, 136-144. doi. org $/ 10.1159 / 000093213$

Chan, J., Guan, X., Ni, Y., Luo, L., Yang, L., Zhang, P., ... Chen, Y. (2017). Dopamine D1-like receptor in lateral habenula nucleus affects contextual fear memory and longterm potentiation in hippocampal CA1 in rats. Behavioral Brain Research, 321, 61-68. Doi: https://doi:10.1016/j. bbr.2016.12.026

Chang, C., Li, N., Wu, Y., Geng, N., Ge, S., ... Wang, J. (2012). Associations between bilateral subthalamic nucleus deep brain stimulation (STN-DBS) and anxiety in Parkinson's disease patients: a controlled study. Journal of 
Neuropsychiatry \& Clinical Neurosciences, 24, 316-325. Doi: https://doi:10.1176/appi.neuropsych.11070170

Choudhury, T. K., Davidson, J. E., Viswanathan, A., \& Strutt, A. M. (2017). Deep brain stimulation of the anterior limb of the internal capsule for treatment of therapy-refractory obsessive compulsive disorder (OCD): a case study highlighting neurocognitive and psychiatric changes. Neurocase, 1-8. Doi: https://doi:10.1080/13554794.2017.1319958

Cif, L., \& Coubes, P. (2017). Historical developments in children's deep brain stimulation. European Journal of Paediatric Neurology, 21, 109-117. Doi: https://doi:10.1016/j. ejpn.2016.08.010

Clark, C. R., Galletly, C. A., Ash, D. J., Moores, K. A., Penrose, R. A., \& McFarlane, A. C. (2009). Evidence-based medicine evaluation of electrophysiological studies of the anxiety disorders. Clinical EEG and Neuroscience, 40, 84-112. Doi: https://doi/pdf/10.1177/155005940904000208

Coenen, V. A., Schlaepfer, T. E., Goll, P., Reinacher, P. C., Voderholzer, U., Tebartz van, E. L., ... Freyer, T. (2016). The medial forebrain bundle as a target for deep brain stimulation for obsessive-compulsive disorder. CNS Spectrums, 1-8. Doi: https://doi:10.1017/S1092852916000286

Cruccu, G., Garcia-Larrea, L., Hansson, P., Keindl, M., Lefaucheur, J. P., ... Paulus, W. (2016). EAN guidelines on central neurostimulation therapy in chronic pain conditions. European Journal of Neurology, 23, 1489-1499. Doi: https://doi:10.1111/ene.13103

Cukiert, A., \& Lehtimaki, K. (2017). Deep brain stimulation targeting in refractory epilepsy. Epilepsia, 58, Supplement 1, 80-84. Doi: https://doi:10.1111/epi.13686

Dalkilic, E. B. (2017). Neurostimulation Devices Used in Treatment of Epilepsy. Current Treatment. Options in Neurology, $19,7$.

Dell'Osso, B., Cremaschi, L., Oldani, L., \& Carlo, A. A. (2017). New Directions in the Use of Brain Stimulation Interventions in Patients with Obsessive-Compulsive Disorder. Current Medicinal Chemistry. Doi: https://doi:10.2174/092986 7324666170505113631

Dos Santos, L., De Andrade, T. G., \& Graeff, F. G. (2010). Social separation and diazepam withdrawal increase anxiety in the elevated plus-maze and serotonin turnover in the median raphe and hippocampus. Journal of Psychopharmacology, 24, 725-731. Doi: https://doi:10.1177/0269881109106954

Dupre, D. A., Tomycz, N., Oh, M. Y., \& Whiting, D. (2015). Deep brain stimulation for obesity: past, present, and future targets. Neurosurgical Focus, 38, E7. Doi: https:// doi:10.3171/2015.3.FOCUS1542

Faria, M. A. (2013). Violence, mental illness, and the brain - A brief history of psychosurgery: Part 3 - From deep brain stimulation to amygdalotomy for violent behavior, seizures, and pathological aggression in humans. Surgical Neurology International, 4, 91. Doi: https://doi:10.4103/21527806.115162
Faria, R., Magalhaes, A., Monteiro, P. R., Gomes-Da-Silva, J., Amelia, T. M., \& Summavielle, T. (2006). MDMA in adolescent male rats: decreased serotonin in the amygdala and behavioral effects in the elevated plus-maze test. Annals of the New York Academy of Science, 1074, 643-649. Doi: http://doi.org/10.1196/annals.1369.062

Fukaya, C., Watanabe, M., Kobayashi, K., Oshima, H., Yoshino, A., \& Yamamoto, T. (2017). Predictive Factors for Long-term Outcome of Subthalamic Nucleus Deep Brain Stimulation for Parkinson's Disease. Neurologia medicochirurgica (Tokyo), 57, 166-171. Doi: https://doi:10.2176/ nmc.oa.2016-0114

Gill, M. J., Ghee, S. M., Harper, S. M., \& See, R. E. (2013). Inactivation of the lateral habenula reduces anxiogenic behavior and cocaine seeking under conditions of heightened stress. Pharmacology, Biochemistry and Behavior, 111, 2429. Doi: https://doi:10.1016/j.pbb.2013.08.002

Golden, S. A., Heshmati, M., Flanigan, M., Christoffel, D. J., Guise, K., ... Pfau, M. L. (2016). Basal forebrain projections to the lateral habenula modulate aggression reward. Nature, 534, 688-692. Doi: https://doi:10.1038/nature18601

Han, B., Jin, H. J., Song, M. Y., Wang, T., \& Zhao, H. (2014). A potential target for the treatment of Parkinson's disease: effect of lateral habenula lesions. Parkinsonism and Related Disorders, 20,1191-1195. Doi: https://doi:10.1016/j. parkreldis.2014.08.022

Harat, M., Rudas, M., Zielinski, P., Birska, J., \& Sokal, P. (2015). Deep Brain Stimulation in Pathological Aggression. Stereotactic and Functional Neurosurgery, 93, 310-315. Doi: https://doi:10.1159/000431373

Heldt, S. A., \& Ressler, K. J. (2006). Lesions of the habenula produce stress- and dopamine-dependent alterations in prepulse inhibition and locomotion. Brain Research, 1073-1074, 229-239. Doi: http://doi.org/10.1016/j. brainres.2005.12.053

Hennigan, K., D'Ardenne, K., \& McClure, S. M. (2015). Distinct midbrain and habenula pathways are involved in processing aversive events in humans. Journal of Neurosciences, 35, 198-208. Doi: https://doi:10.1523/JNEUROSCI.0927-14.2015

Hikosaka, O., Sesack, S. R., Lecourtier, L., \& Shepard, P. D. (2008). Habenula: crossroad between the basal ganglia and the limbic system. Journal of Neurosciences, 28, 11825-11829. Doi: https://doi:10.1523/JNEUROSCI.3463-08.2008

Hong, S., \& Hikosaka, O. (2008). The globus pallidus sends reward-related signals to the lateral habenula. Neuron, 60, 720-729. Doi: https://doi:10.1016/j.neuron.2008.09.035

Howland, R. H. (2013). Deep brain stimulation and aggression. Journal of Neurosurgery, 119, 273-275. Doi: https:// doi:10.3171/2013.1.JNS122308

Jean-Richard Dit, B. P., \& McNally, G. P. (2014). The role of the lateral habenula in punishment. PLOS ONE, 9, e111699. Doi: https://doi:10.1371/journal.pone.0111699 
John, C. S., \& Currie, P. J. (2012). N-arachidonoyl-serotonin in the basolateral amygdala increases anxiolytic behavior in the elevated plus maze. Behavioral Brain Research, 233, 382-388. Doi: https://doi:10.1016/j.bbr.2012.05.025

Kim, J. H., Chang, W. S., Jung, H. H., \& Chang, J. W. (2015). Effect of Subthalamic Deep Brain Stimulation on Levodopa-Induced Dyskinesia in Parkinson's Disease. Yonsei Medical Journal, 56, 1316-1321. Doi: https://doi:10.3349/ ymj.2015.56.5.1316

Kim, Y., Morath, B., Hu, C., Byrne, L. K., Sutor, S. L., Frye, M. A., ... Tye, S. J. (2016). Antidepressant actions of lateral habenula deep brain stimulation differentially correlate with CaMKII/GSK3/AMPK signaling locally and in the infralimbic cortex. Behavioral Brain Research, 306, 170-177. Doi: https://doi:10.1016/j.bbr.2016.02.039

Klinger, N. V., \& Mittal, S. (2016). Clinical efficacy of deep brain stimulation for the treatment of medically refractory epilepsy. Clinical Neurology and Neurosurgery, 140, 11-25. Doi: https://doi:10.1016/j.clineuro.2015.11.009

Krishna, V., Sammartino, F., King, N. K., So, R. Q., \& Wennberg, R. (2016). Neuromodulation for Epilepsy. Neurosurgery Clinics of North America, 27, 123-131. Doi: https:// doi:10.1016/j.nec.2015.08.010

Lecca, S., Meye, F. J., \& Mameli, M. (2014). The lateral habenula in addiction and depression: an anatomical, synaptic and behavioral overview. European Journal of Neuroscience, 39, 1170-1178. Doi: https://doi:10.1111/ejn.12480

Lecourtier, L., Deschaux, O., Arnaud, C., Chessel, A., Kelly, P. H., \& Garcia, R. (2006). Habenula lesions alter synaptic plasticity within the fimbria-accumbens pathway in the rat. Neuroscience, 141, 1025-1032. Doi: https://doi. org/10.1016/j.neuroscience.2006.04.018

Lecourtier, L., Neijt, H. C., \& Kelly, P. H. (2004). Habenula lesions cause impaired cognitive performance in rats: implications for schizophrenia. European Journal of Neuroscience, 19, 2551-2560. doi.org/10.1111/j.0953-816X.2004.03356.x

Li, J., Zuo, W., Fu, R., Xie, G., Kaur, A., ... Ye, J. H. (2016). High Frequency Electrical Stimulation of Lateral Habenula Reduces Voluntary Ethanol Consumption in Rats. International Journal of Neuropsychopharmacology, pyw050. Doi: https://doi:10.1093/ijnp/pyw050

Li, Y., Wang, Y., Xuan, C., Li, Y., Piao, L., ... Zhao, H. (2017). Role of the Lateral Habenula in Pain-Associated Depression. Frontiers in Behaioral Neuroscience, 11, 31. Doi: https://doi:10.3389/fnbeh.2017.00031

Lim, L. W., Prickaerts, J., Huguet, G., Kadar, E., Hartung, H., Temel, Y. (2015). Electrical stimulation alleviates depressive-like behaviors of rats: investigation of brain targets and potential mechanisms. Transational Psychiatry, 5, e535. Doi: https://doi:10.1038/tp.2015.24

Lin, D., \& Parsons, L. H. (2002). Anxiogenic-like effect of serotonin(1B) receptor stimulation in the rat elevated plusmaze. Pharmacology, Biochemistry and Behavior, 71, 581587. Doi: https://doi.org/10.1016/S0091-3057(01)00712-2
Lumsden, D. E., Kaminska, M., Ashkan, K., Selway, R., \& Lin, J. P. (2017). Deep brain stimulation for childhood dystonia: Is 'where' as important as in 'whom'? European Journal of Paediatric Neurology, 21, 176-184. Doi: https:// doi:10.1016/j.ejpn.2016.10.002

Maisonnette, S., Morato, S., \& Brandao, M. L. (1993). Role of resocialization and of 5-HT1A receptor activation on the anxiogenic effects induced by isolation in the elevated plus-maze test. Physiology and Behavior, 54, 753-758. Doi: https://doi.org/10.1016/0031-9384(93)90087-V

Margolis, E. B., \& Fields, H. L. (2016). Mu Opioid Receptor Actions in the Lateral Habenula. PLOS ONE, 11, e0159097. Doi: https://doi:10.1371/journal.pone.0159097

Moraes, C. L., Bertoglio, L. J., \& Carobrez, A. P. (2008). Interplay between glutamate and serotonin within the dorsal periaqueductal gray modulates anxiety-related behavior of rats exposed to the elevated plus-maze. Behavioral Brain Research, 194, 181-186. Doi: https://doi:10.1016/j. bbr.2008.07.005

Moreines, J. L., Owrutsky, Z. L., \& Grace, A. A. (2017). Involvement of Infralimbic Prefrontal Cortex but not Lateral Habenula in Dopamine Attenuation After Chronic Mild Stress. Neuropsychopharmacology, 42, 904-913. Doi: https://doi:10.1038/npp.2016.249

Motta, V., Maisonnette, S., Morato, S., Castrechini, P., \& Brandao, M. L. (1992). Effects of blockade of 5-HT2 receptors and activation of 5-HT1A receptors on the exploratory activity of rats in the elevated plus-maze. Psychopharmacology (Berl), 107, 135-139.

Mulders, A. E. P., Plantinga, B. R., Schruers, K., Duits, A., Janssen, M. L. F., Ackermans, L., ... Temel, Y. (2016). Deep brain stimulation of the subthalamic nucleus in obsessive-compulsive disorder: Neuroanatomical and pathophysiological considerations. European Neuropsychopharmacology, 26, 1909-1919. Doi: https://doi:10.1016/j.euroneuro.2016.10.011

Murphy, C. A., DiCamillo, A. M., Haun, F., \& Murray, M. (1996). Lesion of the habenular efferent pathway produces anxiety and locomotor hyperactivity in rats: a comparison of the effects of neonatal and adult lesions. Behavioral Brain Research, 81, 43-52. Doi: https://doi.org/10.1016/ S0166-4328(96)00041-1

Murrow, R. W. (2014). Penfield's Prediction: A Mechanism for Deep Brain Stimulation. Frontiers in Neurology, 5, 213. Doi: https://doi:10.3389/fneur.2014.00213

Nicolaidis, S. (2017). Neurosurgery of the future: Deep brain stimulations and manipulations. Metabolism, 69S, S16-S20. Doi: https://doi:10.1016/j.metabol.2017.01.013

Ootsuka, Y., \& Mohammed, M. (2015). Activation of the habenula complex evokes autonomic physiological responses similar to those associated with emotional stress. Physiological Reports, 3. Doi: https://doi:10.14814/phy2.12297

Ostrem, J. L., San, L. M., Dodenhoff, K. A., Ziman, N., Markun, L. C., Racine, C. A., ... Starr, P. A. (2017). Subthalamic 
nucleus deep brain stimulation in isolated dystonia: A 3-year follow-up study. Neurology, 88, 25-35. Doi: https:// doi:10.1212/WNL.0000000000003451

Paxinos, G., \& Watson, C. (2006). The Rat Brain in Stereotaxic Coordinates: Hard Cover Edition. Elsevier Science.

Plotkin, R. (1982). Results in 60 cases of deep brain stimulation for chronic intractable pain. Applied Neurophysiology, 45, 173-178.

Pobbe, R. L., \& Zangrossi, H., Jr. (2010). The lateral habenula regulates defensive behaviors through changes in 5-HTmediated neurotransmission in the dorsal periaqueductal gray matter. Neuroscience Letters, 479, 87-91. Doi: https:// doi:10.1016/j.neulet.2010.05.021

Ray, C. D., \& Burton, C. V. (1980). Deep brain stimulation for severe, chronic pain. Acta Neurochirurgica Supplement (Wien.), 30, 289-293.

Rolls, E. T. (2017). The roles of the orbitofrontal cortex via the habenula in non-reward and depression, and in the responses of serotonin and dopamine neurons. Neuroscience \& Biobehavioral Reviews, 75, 331-334. Doi: https:// doi:10.1016/j.neubiorev.2017.02.013

Rosenow, J. M., Mogilnert, A. Y., Ahmed, A., \& Rezai, A. R. (2004). Deep brain stimulation for movement disorders. Neurological Research, 26, 9-20. Doi: https:// doi:10.1179/016164104773026480

Roth, R. M., Flashman, L. A., Saykin, A. J., \& Roberts, D. W. (2001). Deep brain stimulation in neuropsychiatric disorders. Current Psychiatry Reports, 3, 366-372.

Salgado-Lopez, L., Pomarol-Clotet, E., Roldan, A., Rodriguez, R., Molet, J., ... Sarro, S. (2016). Letter to the Editor: Deep brain stimulation for schizophrenia. Journal of Neurosurgery, 125, 229-230. Doi: https://doi:10.3171/2015.12. JNS152874

Schwalb, J. M., \& Hamani, C. (2008). The history and future of deep brain stimulation. Neurotherapeutics, 5, 3-13. Doi: https://doi:10.1016/j.nurt.2007.11.003

Setem, J., Pinheiro, A. P., Motta, V. A., Morato, S., \& Cruz, A. P. (1999). Ethopharmacological analysis of 5-HT ligands on the rat elevated plus-maze. Pharmacology, Biochemistry and Behavior, 62, 515-521. doi.org/10.1016/S00913057(98)00193-2

Shelton, L., Becerra, L., \& Borsook, D. (2012). Unmasking the mysteries of the habenula in pain and analgesia. Progress in Neurobiology, 96, 208-219. Doi: https://doi:10.1016/j. pneurobio.2012.01.004

Song, M., Jo, Y. S., Lee, Y. K., \& Choi, J. S. (2017). Lesions of the lateral habenula facilitate active avoidance learning and threat extinction. Behavioral Brain Research, 318, 12-17. Doi: https://doi:10.1016/j.bbr.2016.10.013

Sourani, D., Eitan, R., Gordon, N., \& Goelman, G. (2012). The habenula couples the dopaminergic and the serotonergic systems: application to depression in Parkinson's disease.
European Journal of Neuroscience, 36, 2822-2829. Doi: https://doi:10.1111/j.1460-9568.2012.08200.x

Sturm, V., Lenartz, D., Koulousakis, A., Treuer, H., Herholz, K., Klein, J. C., ... Klosterkötter, J. (2003). The nucleus accumbens: a target for deep brain stimulation in obsessivecompulsive- and anxiety-disorders. Journal of Chemical. Neuroanatomy, 26, 293-299. doi.org/10.1016/j.jchemneu.2003.09.003

Thornton, E. W., \& Bradbury, G. E. (1989). Effort and stress influence the effect of lesion of the habenula complex in one-way active avoidance learning. Physiology \& Behavior, 45, 929-935. doi.org/10.1016/0031-9384(89)90217-5

Toda, H., Saiki, H., Nishida, N., \& Iwasaki, K. (2016). Update on Deep Brain Stimulation for Dyskinesia and Dystonia: A Literature Review. Neurologia Medico-Chirurgica (Tokyo), 56, 236-248. Doi: https://doi:10.2176/nmc.ra.2016-0002

Udupa, K., \& Chen, R. (2015). The mechanisms of action of deep brain stimulation and ideas for the future development. Progress in Neurobiology, 133, 27-49. Doi: https:// doi:10.1016/j.pneurobio.2015.08.001

Vadovicova, K. (2014). Affective and cognitive prefrontal cortex projections to the lateral habenula in humans. Frontiers in Human Neuroscience, 8, 819. Doi: https:/doi:10.3389/ fnhum.2014.00819

Velasquez, K. M., Molfese, D. L., \& Salas, R. (2014). The role of the habenula in drug addiction. Frontiers in $\mathrm{Hu}$ man Neurosciences, 8, 174. Doi: https://doi:10.3389/ fnhum.2014.00174

Wickens, A. P., \& Thornton, E. W. (1996). Circling behaviour induced by apomorphine after lesions of the habenula. Experimental Brain Research, 109, 17-21.

Yadid, G., Gispan, I., \& Lax, E. (2013). Lateral habenula deep brain stimulation for personalized treatment of drug addiction. Frontiers in Human Neuroscience, 7, 806. Doi: https:// doi:10.3389/fnhum.2013.00806

Yang, L. M., Hu, B., Xia, Y. H., Zhang, B. L., \& Zhao, H. (2008). Lateral habenula lesions improve the behavioral response in depressed rats via increasing the serotonin level in dorsal raphe nucleus. Behavioral Brain Research, 188, 84-90. Doi: https://doi.org/10.1016/j.bbr.2007.10.022

Yeomans, J. S. (1990). Principles of brain stimulation. New York: Oxford University Press.

Zhao, H., Zhang, B. L., Yang, S. J., \& Rusak, B. (2015). The role of lateral habenula-dorsal raphe nucleus circuits in higher brain functions and psychiatric illness. Behavioral Brain Research, 277, 89-98. Doi: https://doi:10.1016/j. bbr.2014.09.016 